\title{
Multidisciplinary approaches interventions in prevention of childhood obesity: targeted systematic review
}

\author{
Hanan Mohamed Mohamed Tork ${ }^{1} *$ and Thomas Boggatz ${ }^{2}$ \\ ${ }^{I^{*} D e p t . ~ o f ~ P e d i a t r i c ~ H e a l t h ~ N u r s i n g, ~ F a c u l t y ~ o f ~ N u r s i n g, ~ Z a g a z i g ~ U n i v e r s i t y, ~ E g y p t ~}$ \\ ${ }^{2}$ University of Applied Sciences, Salzburg, Austria \\ *Corresponding author E-mail: hotork@zu.edu.eg
}

\begin{abstract}
Among children ages 7-19, about 1 in 3 are overweight and obese (BMI-for-age at or above the 85th percentile of the 2000 CDC growth charts.); $32.1 \%$ of all boys and 31.3\% of all girls are overweight and obese. Because of the dramatic and alarming increase in childhood obesity and its associated health risks, obesity prevention programs targeting children can and should be developed to promote the health of the public. This study is an overview of different interventions conducted, to guide efforts for an effective management of childhood obesity.

The aim of this review was to assess the literature regarding the prevention of childhood obesity. Databases that were accessed for current literature included Medline, Cochrane and CINAHL. A total of 26 articles were found based on the inclusion criteria for this study. Only $38 \%$ of studies required parents' participation, the sample sizes of the studies varied considerably from 201 to 3135 children. $92 \%$ of studies used randomized controlled trials and the range of intervention duration ranged from eight weeks to four years. Implications for future research and practice are presented.
\end{abstract}

Keywords: Childhood obesity, Body Mass Index, intervention, Overweight, Prevention, Weight gain, Systematic reviews

\section{Introduction}

Childhood obesity and overweight are defined by the Centers for Disease Control and Prevention (CDC) as a body mass index (BMI) greater than or equal to the 95th percentile for children and adolescents of the same age and gender $(1,2)$. Obesity/overweight has been declared an epidemic and a "public health crisis" among children worldwide (3). The World Health Organization ranked overweight and obesity as the fifth leading global risk for mortality. In addition, $44 \%$ of the diabetes burden, $23 \%$ of the ischemic heart disease burden and between $7 \%$ and $41 \%$ of certain cancer burdens are attributable to overweight and obesity (4). Childhood obesity is associated with many health risks; it is the leading cause of pediatric hypertension and associated with type 2 diabetes mellitus, orthopedic complications, increased risk of coronary heart disease, and increased stress on weight-bearing joints (5). As a result, these children are challenged with the negative social consequences of overweight and obesity, as well as the psychosocial problems associated with peer differences and rejection, body image, stigma, social isolation, anxiety and depression, and poor social competence and self-esteem that contribute to decreased quality of life $(6,7)$. In addition, the long-term financial health costs associated with obesity are steadily increasing $(8,9)$. Moreover, prevalence levels have been rapidly increasing for more than two decades, in parallel with changes in dietary and physical activity patterns, with an unequal distribution across socioeconomic groups (10). The dramatic increase in the prevalence of childhood overweight and its resultant comorbidities are associated with significant health and financial burdens, warranting strong and comprehensive prevention efforts. Prevention is one of the hallmarks of pediatric practice and includes such diverse activities as newborn screenings, immunizations, and promotion of car safety seats and bicycle helmets (1).

Once children (and adults) are obese, it is often difficult for them to lose weight through physical activity and healthy diet. Preventing weight gain from an early age, i.e. in childhood, is therefore recognized as a strategy that will reap health benefits in the long term (4). When developing and implementing childhood obesity prevention intervention, question arise; should we target children as a whole or focus specially on those children at risk? Ells et al. strongly advocated that extremely difficult to confidently identify all children at risk of developing obesity. It is also true to argue that interventions to promote a healthy diet and increased physical activity will benefit all children, irrespective of obesity risk (11). 
Obesity prevention is a pressing need as healthcare costs rise in the treatment of its complications. Today, most obesity prevention programs target children who are considered high-risk. For example, those who already qualify as obese or those who have obese parents. Studies on the effectiveness of early intervention programs have concluded that the introduction of healthy changes may decrease or prevent the development of obesity and associated health problems in school-aged children (12). Prevention programs include many interventions, from breastfeeding to nutrition programs. The objective of this review was to assess the literature regarding the prevention of childhood obesity.

\subsection{Identification of the problem}

The problem of limited evidence to demonstrate the effectiveness of interventions shaped the aim of the review, which was to summaries and analyze strategies used to enhance the prevention of childhood obesity and overweight.

\section{Methods}

\subsection{Search strategy and Inclusion criteria}

A comprehensive search of the available English language literature from June 2001 to October 2011 was conducted utilizing the Medline, Cochrane and CINAHL (Cumulative Index to Nursing \& Allied Health) databases. In order to maximize the yield of the search, several keyword identifiers were utilized (Table 1). In addition, reference lists were screened to identify additional relevant articles; similar strategies were run in the other databases. Full texts of all reviews identified as potentially eligible were obtained and further screened against the eligibility criteria. Disagreement between reviewers regarding eligibility was resolved via discussion with independent third reviewer.

Studies were selected based on meeting the following criteria: (a) Studies only in English language (b) an obesityrelated intervention, (c) Studies that used subjective measures as the exposure (e.g., using questionnaires only) were excluded as these are more likely to be biased than studies using objective measures (e.g., as physical activity), (d) an age criterion where subjects are between 7 and 19 years. No specific conditions were set for controlled studies. Therefore, excluded all studies whose primary aim was not to intervene on obesity or related health and fitness measures (ie, interventions for the prevention of cardiovascular disease, type II diabetes, etc). In addition, sample sizes less than 100 participants would have limited statistical power to find an effect; thus, four studies were excluded from the review.

\subsection{Data extraction}

One reviewer extracted data from identified studies, which met the inclusion criteria. To describe the characteristics of included studies, the following information was extracted: Author; year of publication, age group, description of intervention, the setting and outcomes.

Table 1: Search strategy in Medline

\begin{tabular}{cl}
\hline 1 & Obesity [Mesh] \\
2 & Overweight OR over weight \\
3 & Weight gain [Mesh] \\
4 & Body Mass Index OR BMI \\
5 & 1 OR 2 OR 3 OR 4 \\
6 & Child OR children OR pediatr* \\
7 & 5 AND 6 \\
8 & Obesity intervention \\
9 & Physical activity \\
10 & Obesity prevention \\
11 & preventive measures \\
12 & Nutrition program \\
13 & Review [Mesh] \\
14 & Exercise \\
15 & Life Style \\
\hline
\end{tabular}




\section{Results}

The searches initially yielded 2100 references and preliminary sift was conducted to remove the obviously irrelevant papers. Duplicate publications were excluded and in all cases of duplicates the first publication was selected for inclusion. This left 1050 records for further screening, which were then imported into an Endnote bibliographic database and following a process of electronic and manual elimination of duplicates; 950 records were treatment program or had a target population outside the selected age group, this lifted 100 potentially eligible papers. After fulltext articles appraisal 74 were excluded and 26 papers were included in this review (Figure1). Selected articles related to prevention are listed in Table 2. Lobstein et al. (3) expressed eloquently that prevention of obesity in childhood is the only feasible option and prevention of overweight is more effective than interventions that target the correction of obesity because of the recalcitrant nature of obesity.

\subsection{The studies characteristics}

A summary of study characteristics is provided in Table 2. All 26 articles on which this systematic review is based were published in peer review journals and are in the English language and no conflict of interest was declared. Of 26 studies, fourteen were conducted in the USA, eight in Western Europe (Belgium, Sweden, Netherlands, two in France and two UK), two in South America (Chile and Brazil), one in Australia, and one in China. One in Intervention durations ranged from eight weeks to four years. One of the interventions reviewed included only girls and 25 interventions included both girls and boys participants. The sample sizes of the studies varied considerably, from 201 children in the study by Neumark-Sztainer et al. (13) to 3135 children in the study by Marcus et al. (14). Regarding the parents participation, sixteen interventions did not include parents and ten required parents' participation. Of the 26 interventions that examined prevention, 24 (92\%) used randomized controlled trials, one (4\%) (15) tested the intervention using a pretestposttest evaluation design with no control group and (4\%) (16) used a community-based participatory research design.

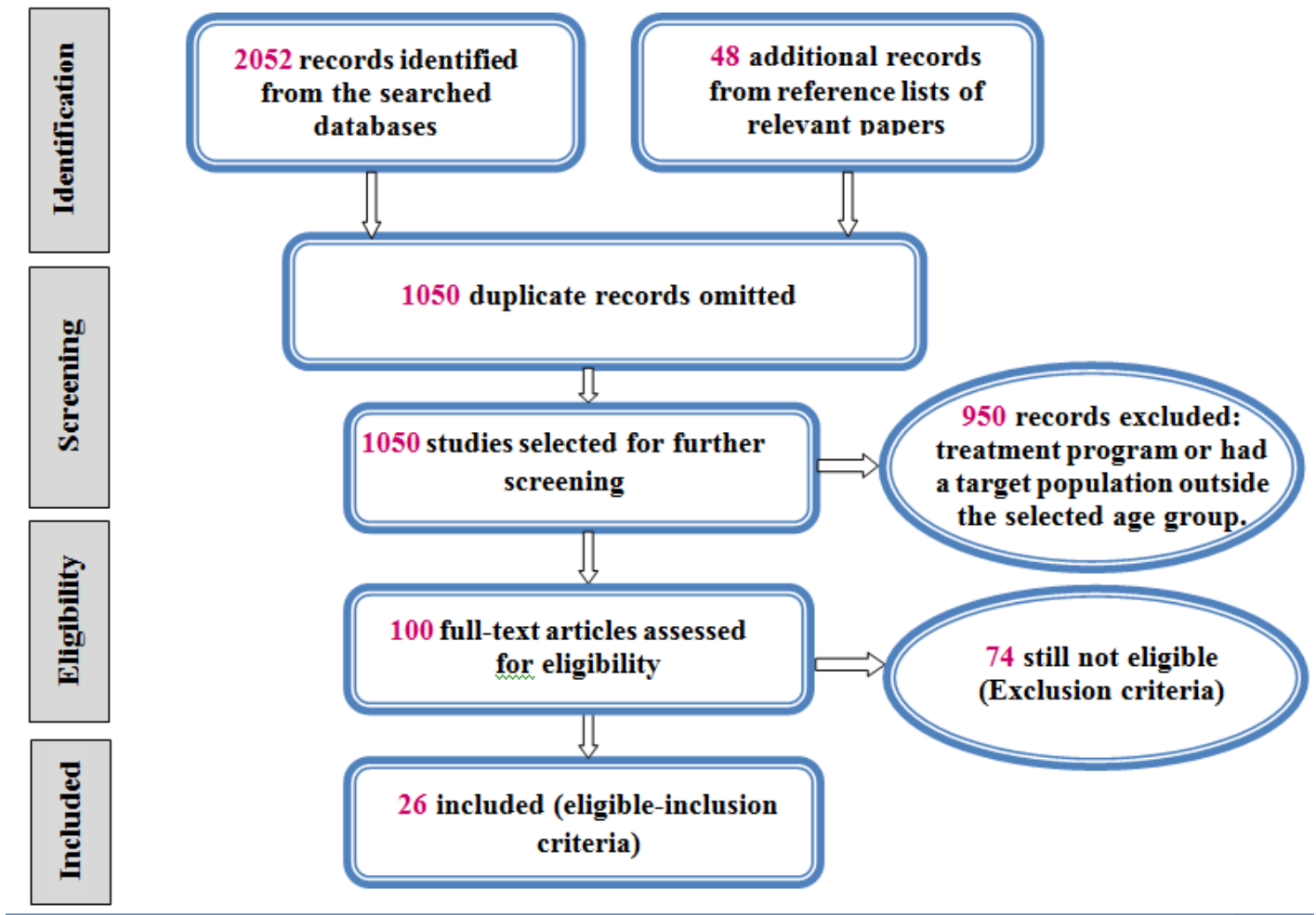

Figure 1: Flowchart for selecting studies 

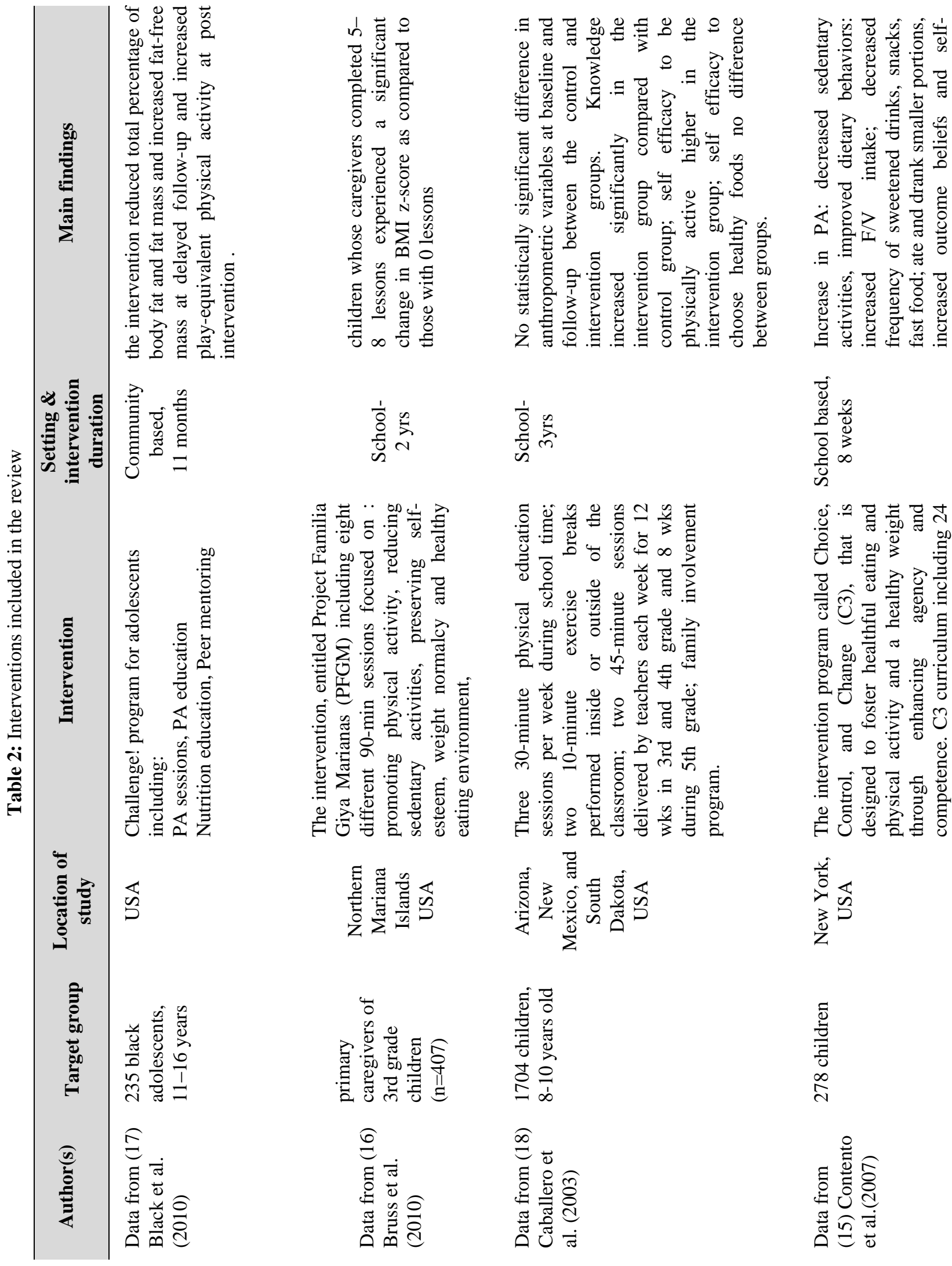

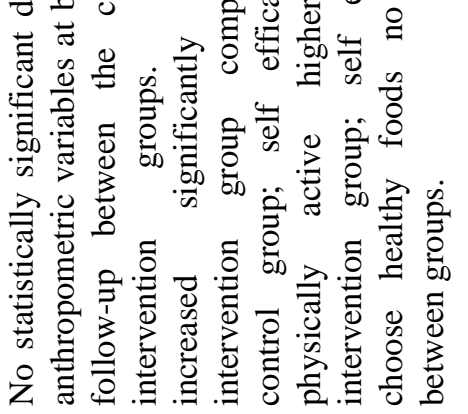

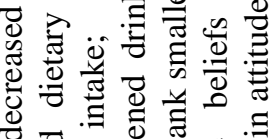

ช

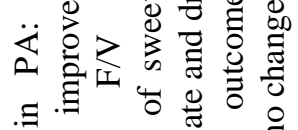

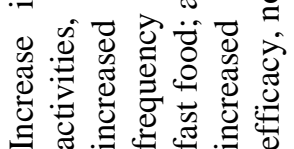

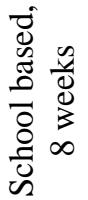

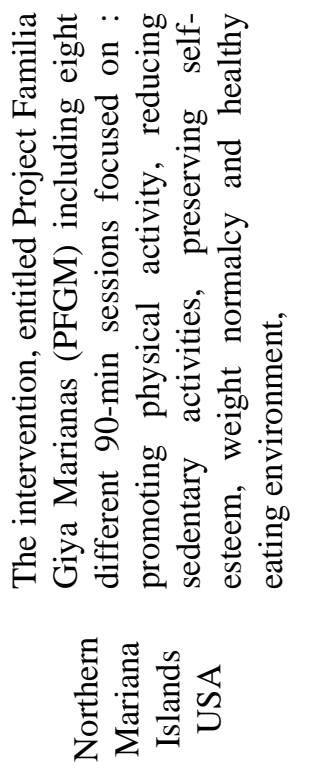

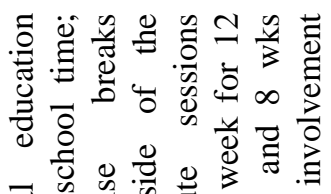

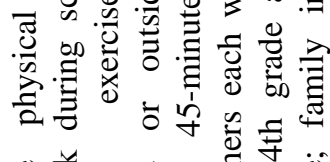

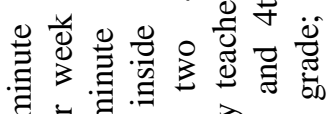

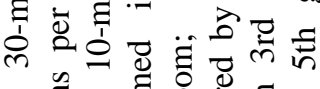

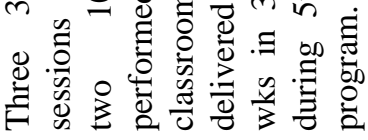

נ.

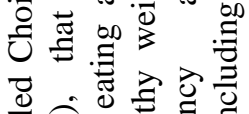

导

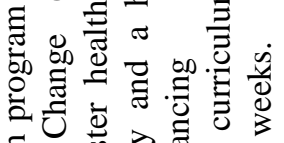
б

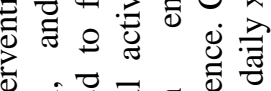
离

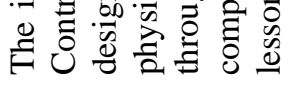

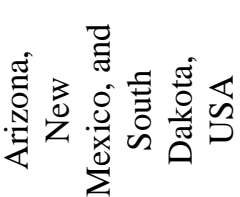
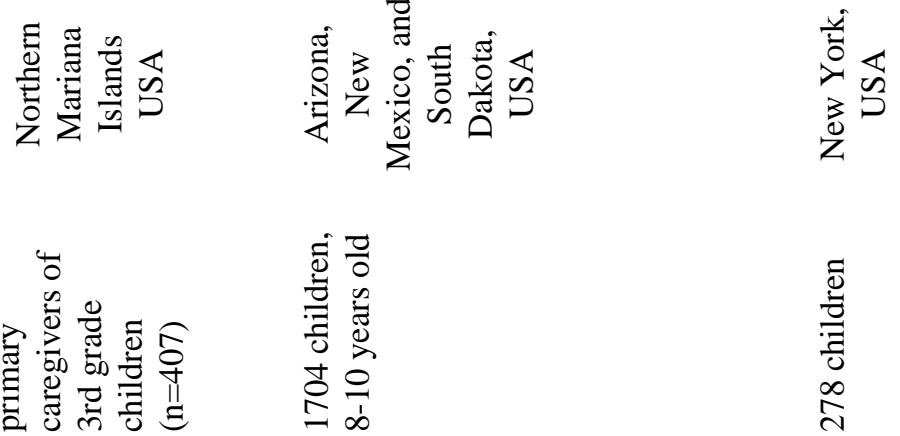

$\frac{0}{0}$
$\frac{0}{0}$
$\frac{0}{0}$
$\frac{1}{2}$

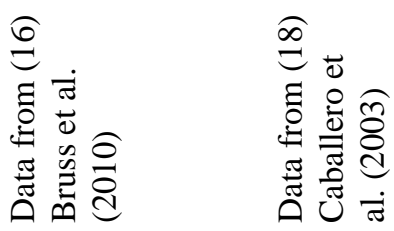

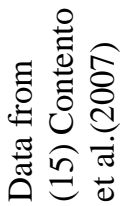




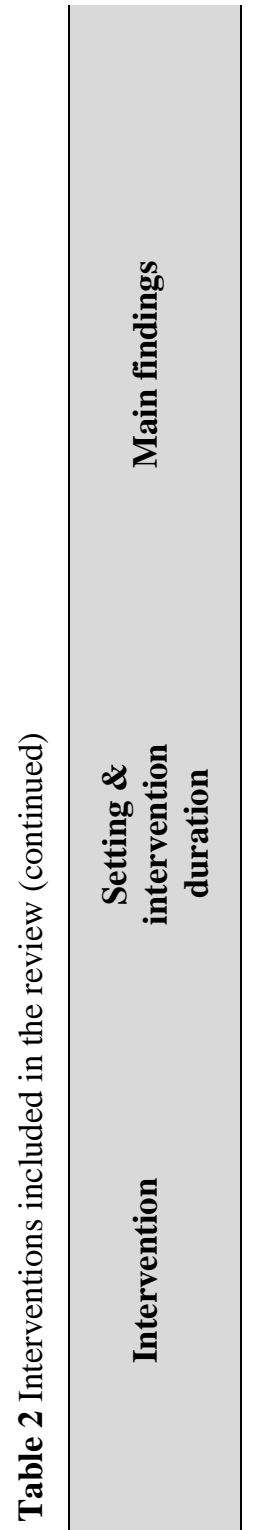

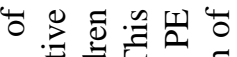

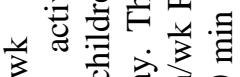

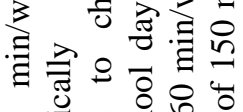

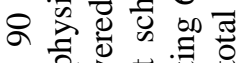

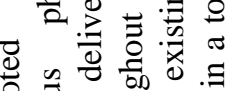

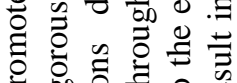
ํ. 。

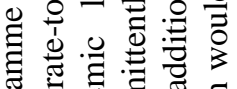

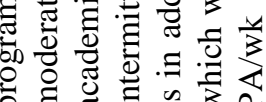

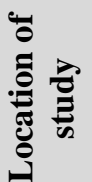

言

高

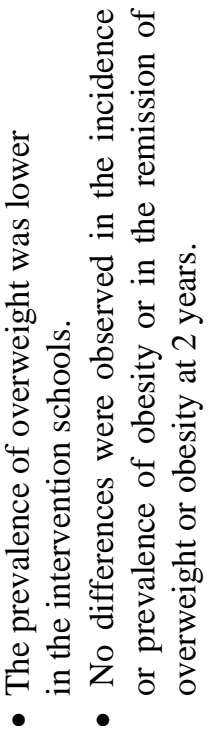

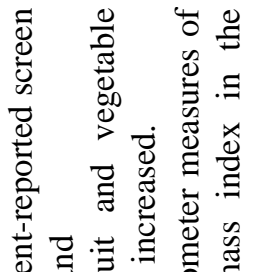

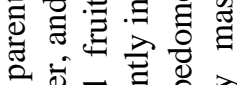

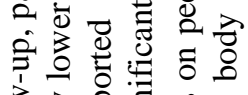

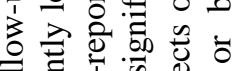

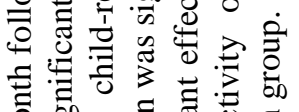

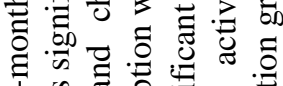
ठิ

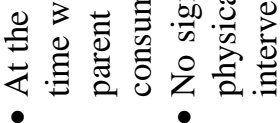

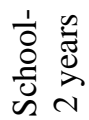

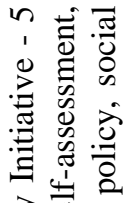

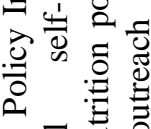

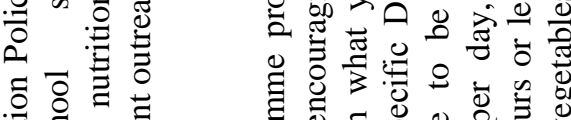

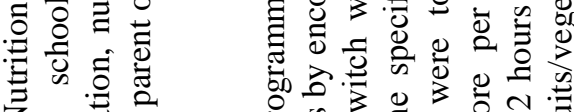

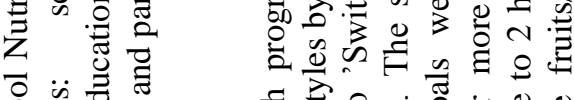

官苍

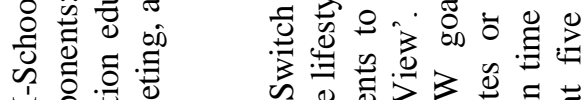

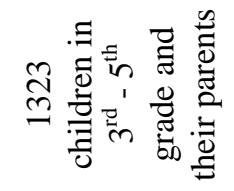

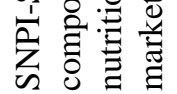

音

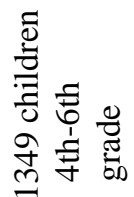

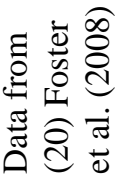

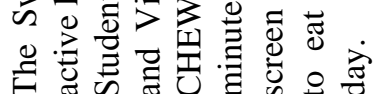

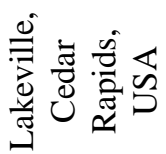

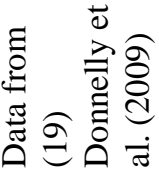

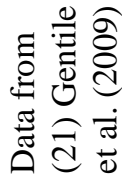

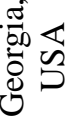

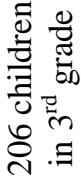

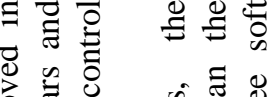

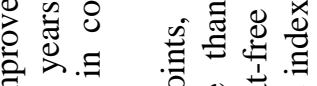

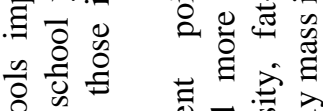

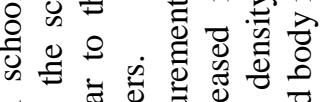

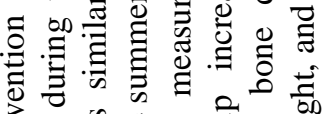

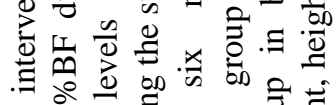

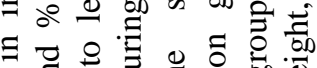
ฮี

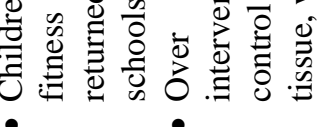

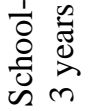

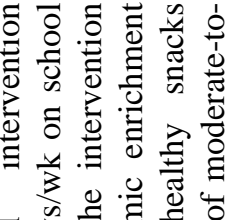

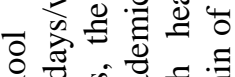

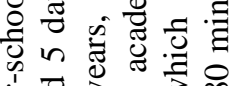
$\frac{1}{4} \square$

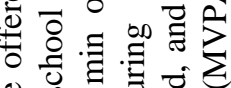

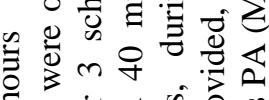

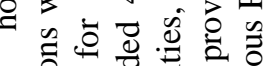

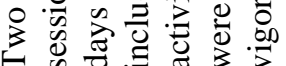

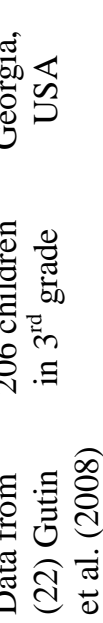




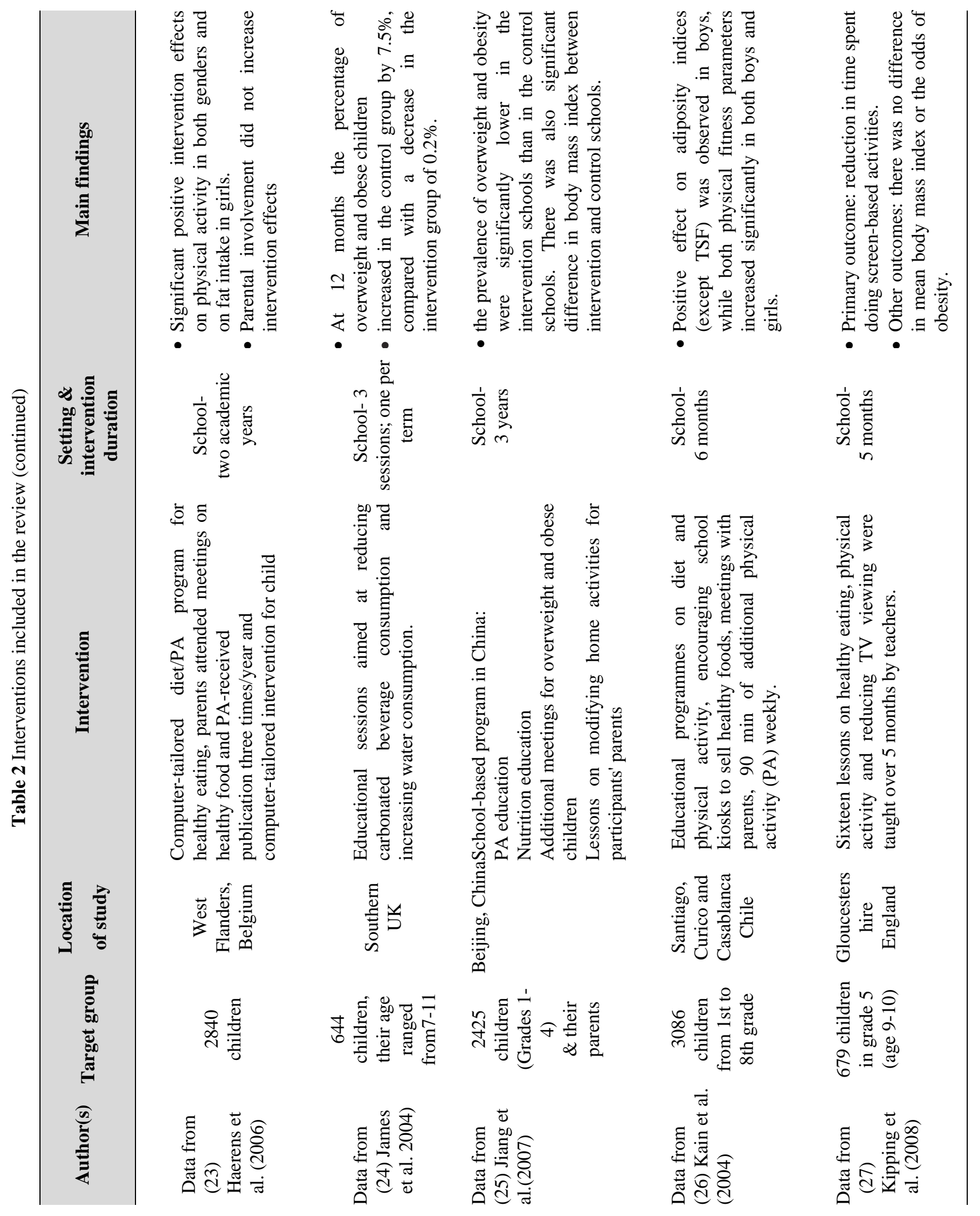



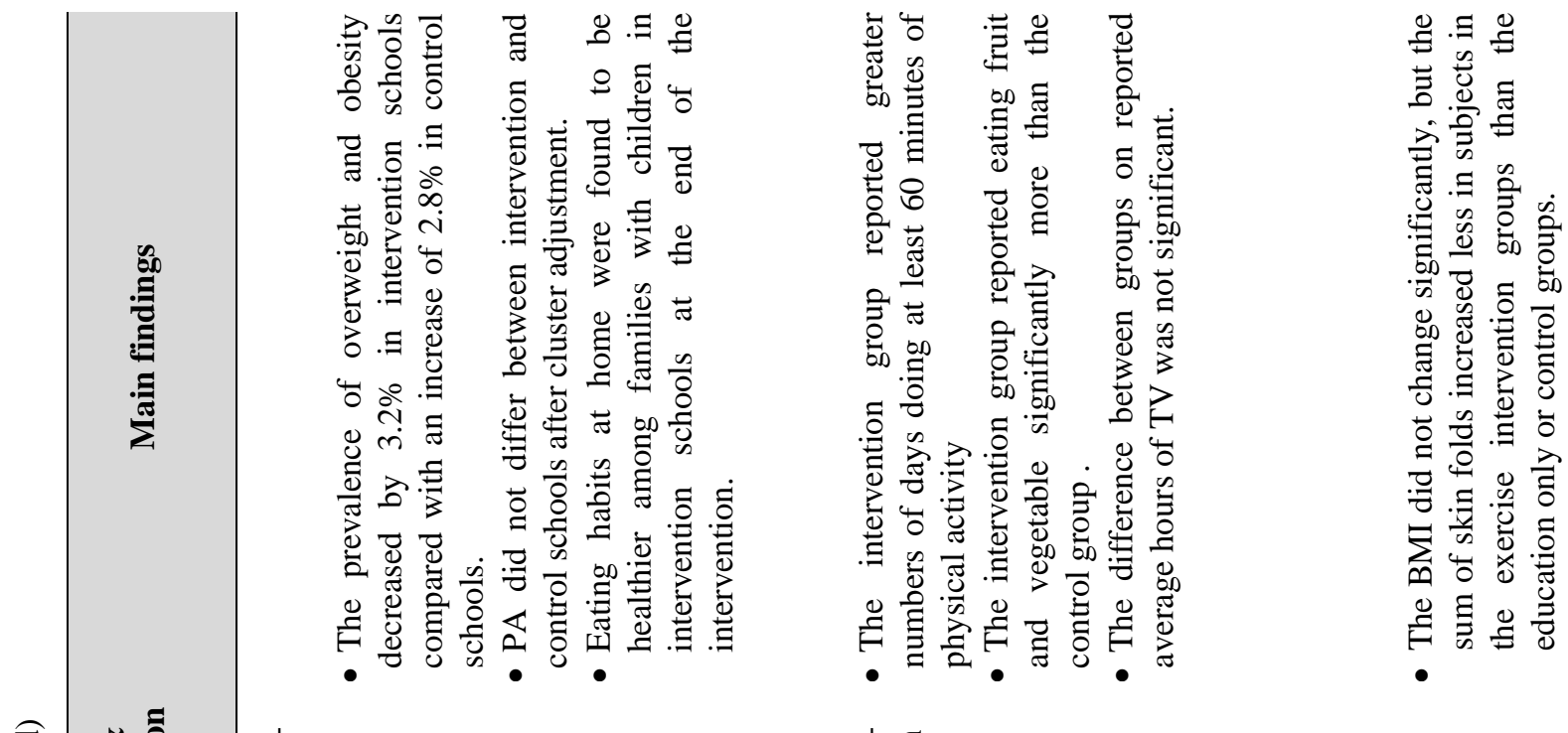

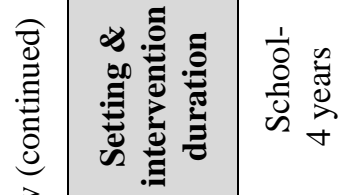

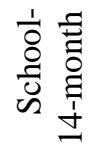

$\begin{array}{ll}1 & n \\ 0 & 0 \\ 0 & 0 \\ 0 & 0 \\ \text { ñ } & \text { d }\end{array}$

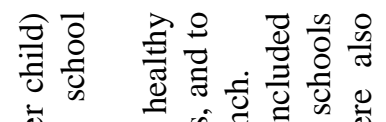

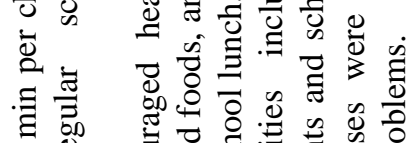

:

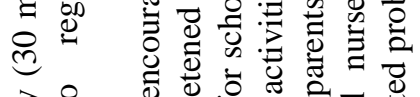

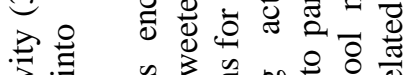

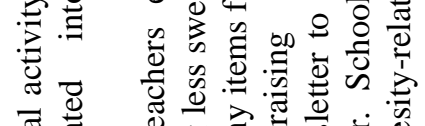

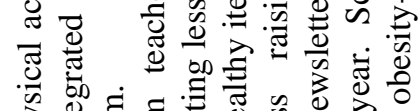

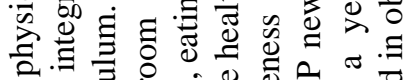

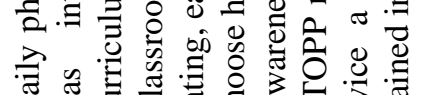

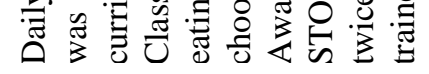

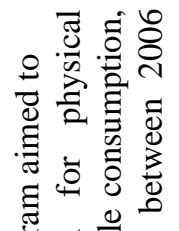

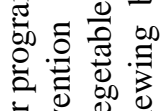

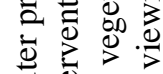

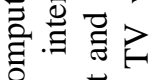

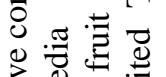

害完

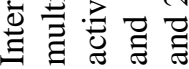

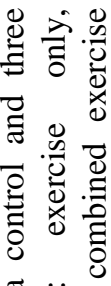

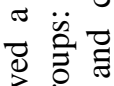

熍

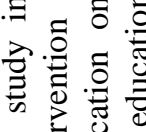

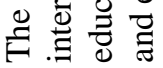

芦

芯

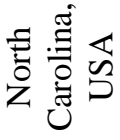

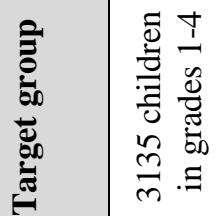

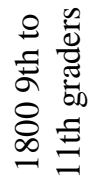

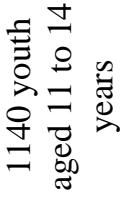

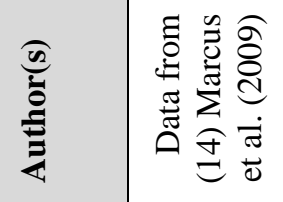

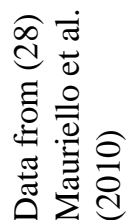

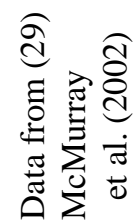




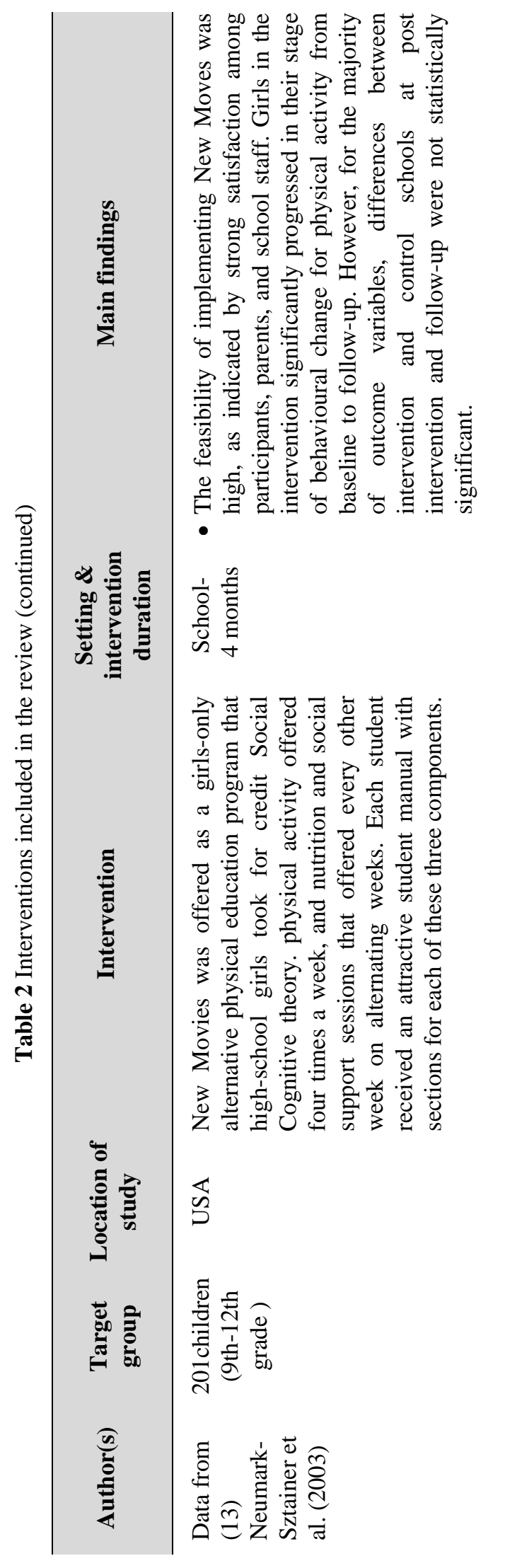

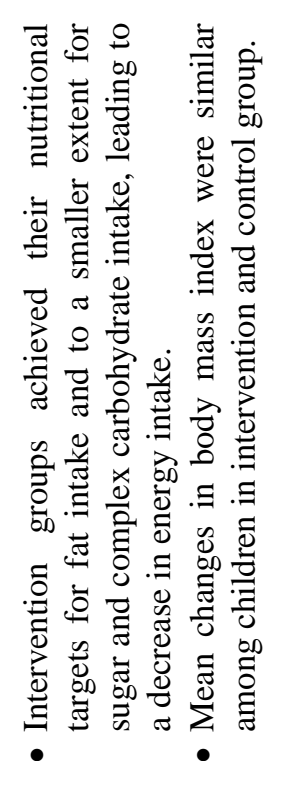

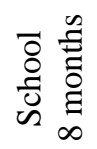
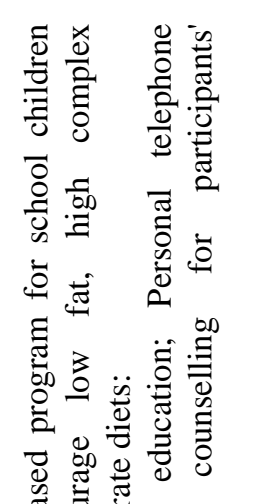

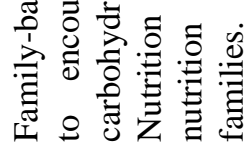

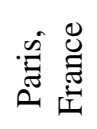

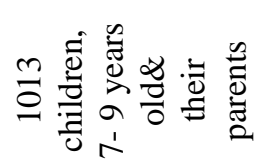

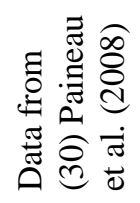

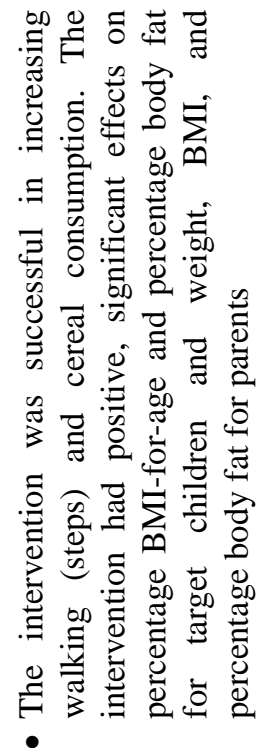

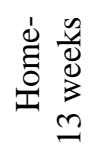

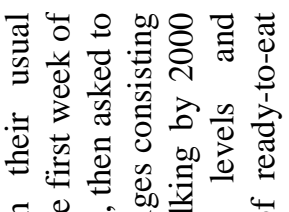
.尹

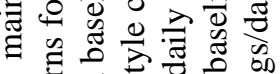

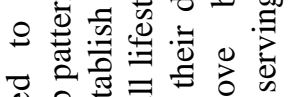

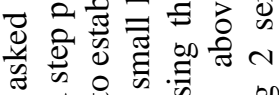

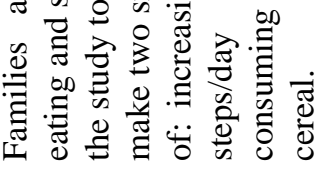

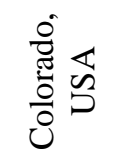

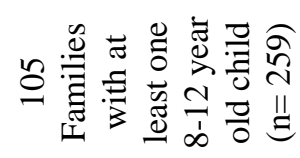

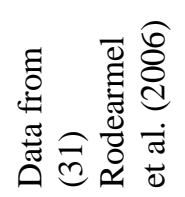




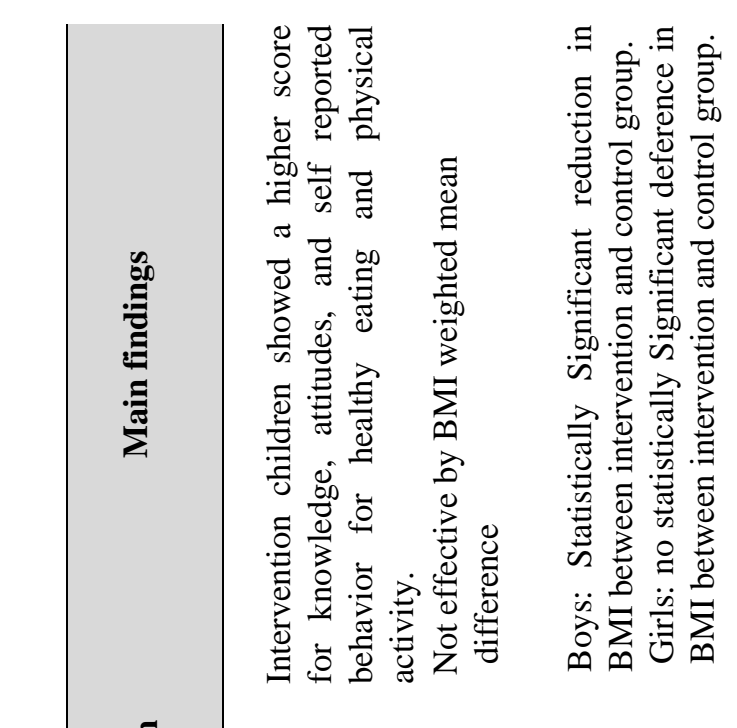

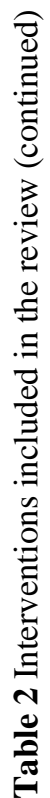

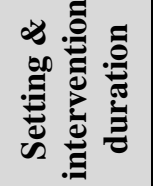

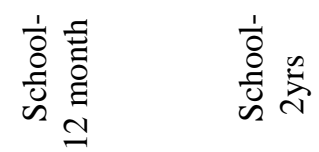

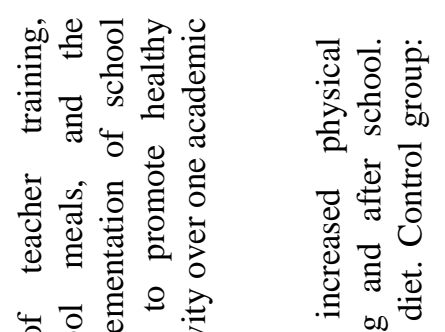

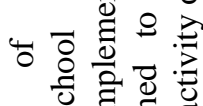

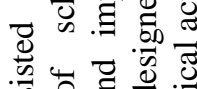

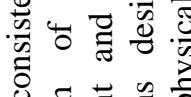

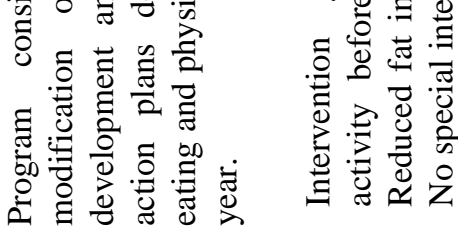

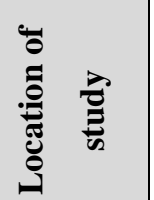

.

$\stackrel{3}{3}$

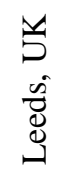

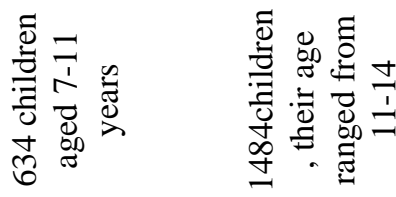

तु बे

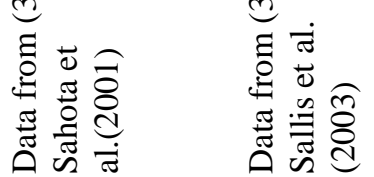

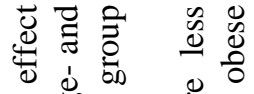

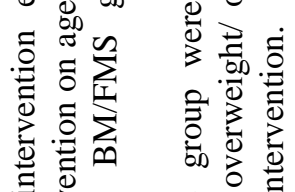

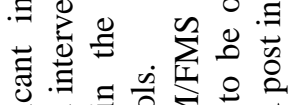

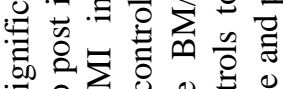

क

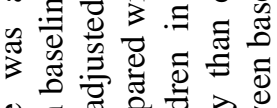

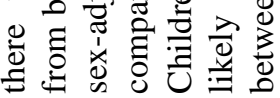

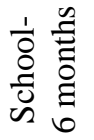

चี

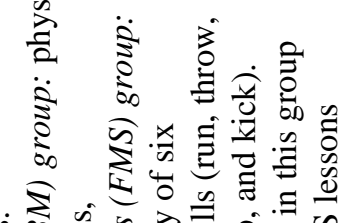

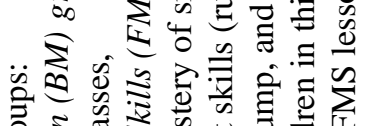

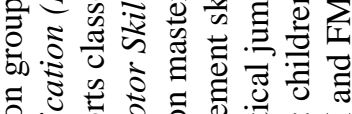

.0ี

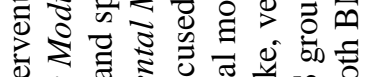

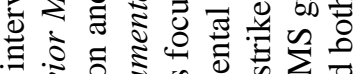

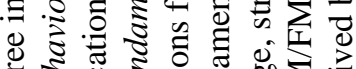

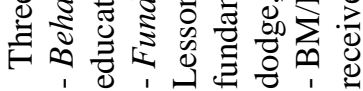

亲:

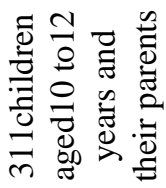

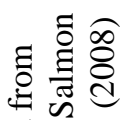

莺穴宁

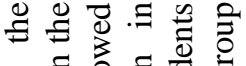

$\Xi . \Xi 00$ 을

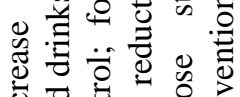

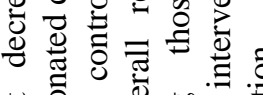

氥

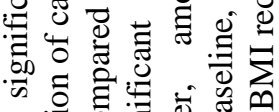

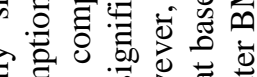

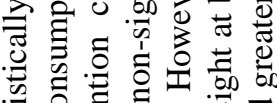

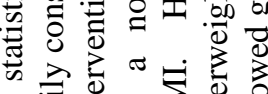

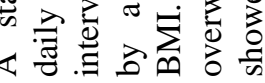

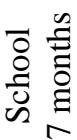

पे

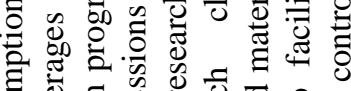

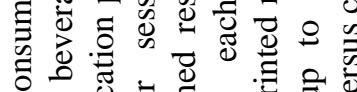

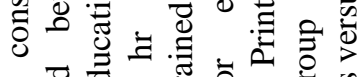

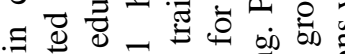

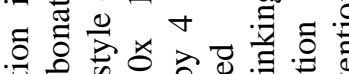

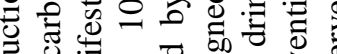

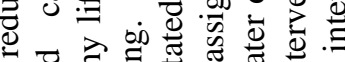

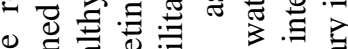

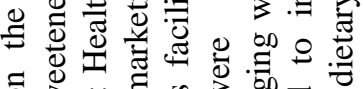
б

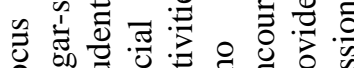

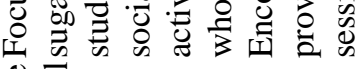
을

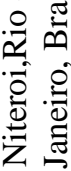

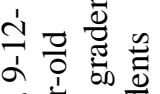
守苋氞胥

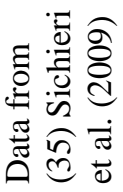




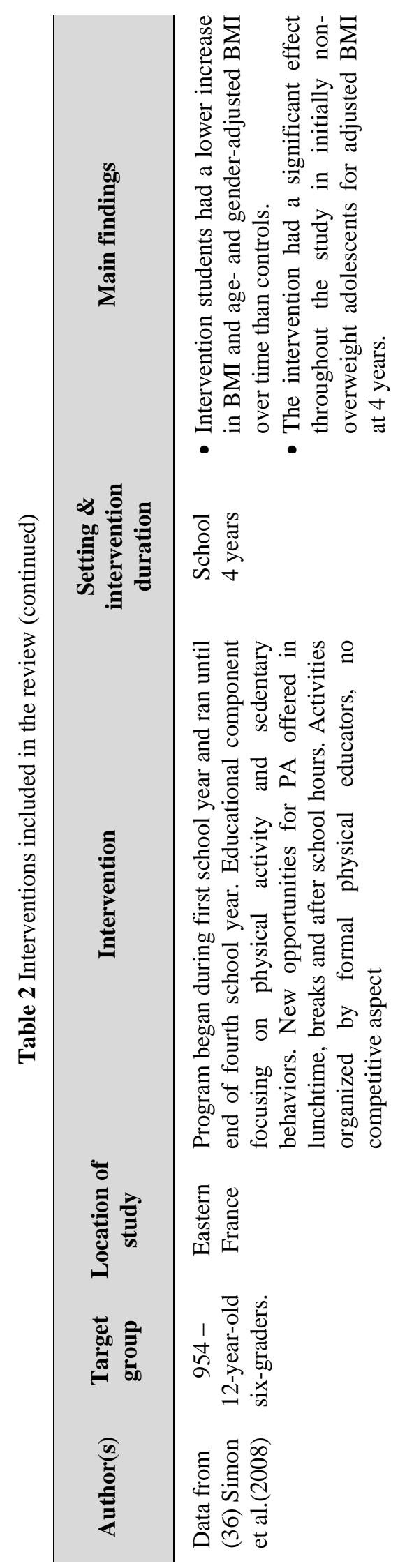

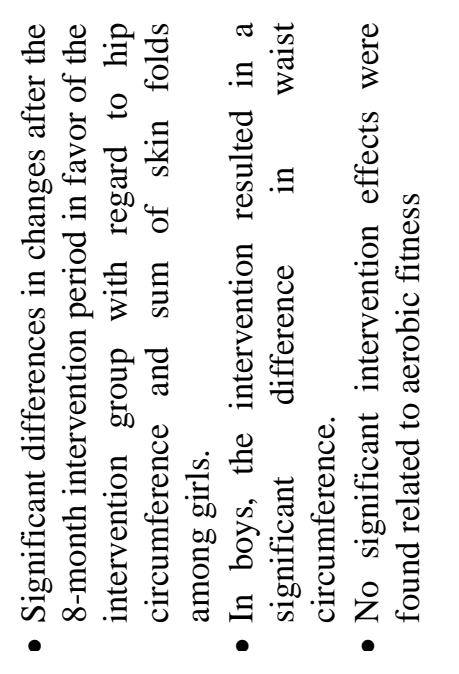

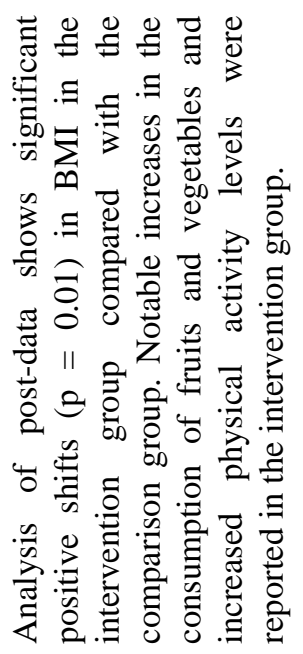

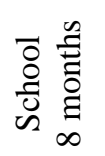

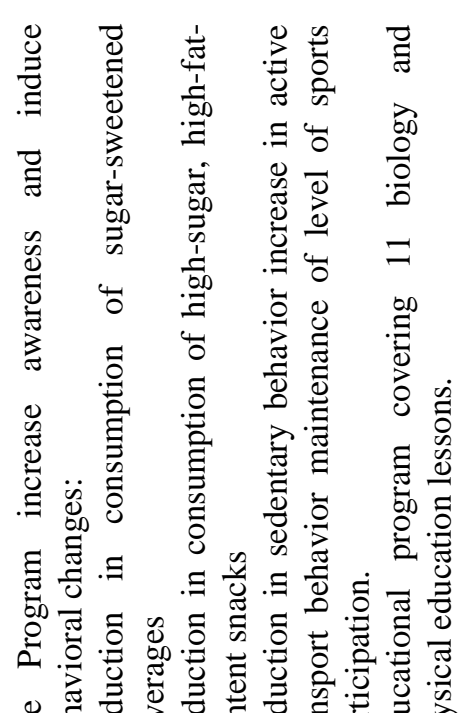

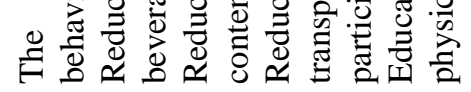
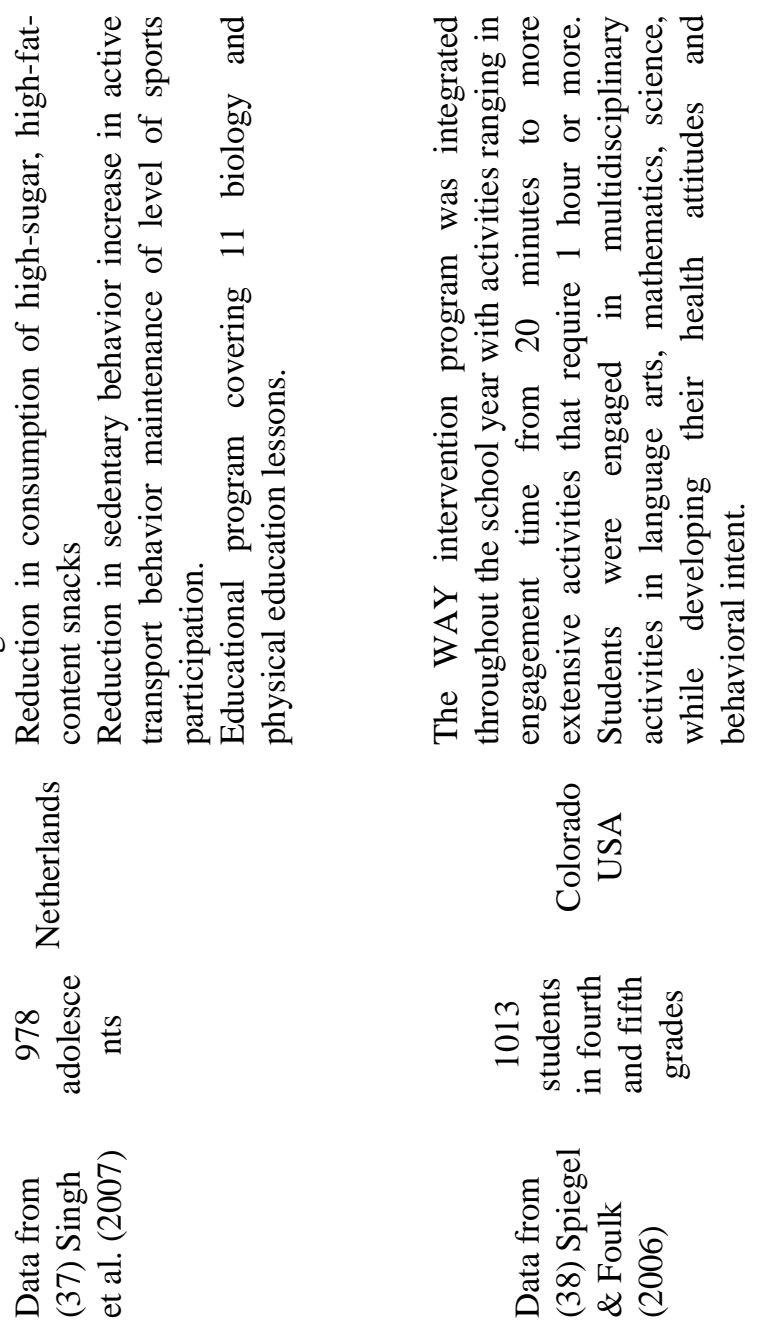


\subsection{Intervention type}

The 26 studies utilized a variety of obesity-related interventions in children (Table 2). Of these interventions, 6 only utilized an implementation or a modification of an existing physical activity program or an in-school physical education class $(18,19,34,36,37,38)$. Another 10 intervention protocols only used health and fitness educational models, dietary regimens, or physical activity behavior modification strategies $(13,16,17,20,23,24,2526,27,30)$. The remaining 10 intervention studies utilized combinations of physical activity programs, health/fitness educational models, and/or dietary/ nutritional regimens $(14,15,21,22,28,29,31,32,33,35)$. Prevention strategies focused on building healthy habits related to nutrition and activity has more stable Long-term results as compared with strategies focused on limiting behaviors. For example, children who were encouraged to increase their fruit and vegetable intake were more likely to demonstrate significant decreases in percentage of overweight than the children who were encouraged to decrease their fat and sugar intake $(30,37,38)$.

\section{Discussion}

In a recent Cochrane review and meta-analysis by Waters et al. although levels of parent participation were not considered in the Cochrane review, Waters et al. conclude that the greater effectiveness seen in non-school settings is likely related to level of parental engagement (39). In addition, a wealth of literature supports the importance of parental involvement in childhood obesity prevention $(40,41,42,43)$. In the current review, Haerens and colleagues found that parents provided greater support after receiving the same computer-tailored intervention as their children. After 1 year, girls had a decreased fat intake and higher levels of physical activity, yet after 2 years, there were no differences with or without parental support. The authors suggested that one weakness of the study was the lack of evaluation of parental involvement. While some interventions emphasized the importance of parent involvement, only $38 \%$ of the reviewed studies required the parent participation. It may be due to that the most of these studies were conducted in school setting or the target population was school age children and adolescents rather than young children (23).

The setting of most intervention described in the included studies (92\%) focused on the schools and only $8 \%$ were conducted in community settings. Nielsen et al., declare that the rationale for working in community settings as the future focus for addressing the childhood obesity epidemic is strong. Adolescents obtain more than $90 \%$ of their total calories from outside the school setting, the majority from within their homes $(60.5 \%)$ or from restaurants $(19.3 \%)(44)$. The authors argue that new directions beyond the central focus on schools must be explored in order to effectively combat the burgeoning obesity among children. Specially that the mixed and modest results of school-centered trials to date argue that this approach alone is not effective in making a real dent in childhood obesity prevention (45). On the other hand, school-based programs have had limited success in engaging parents to change household behavior, and thereby reducing the obesigenic environment at home, with some exceptions (46).

While some of the interventions were long term, there were eight interventions (30\%) had a duration of 6 months or less, which appears to be an inadequate duration to achieve significant changes in BMI. This conclusion is supported by Kain et al. (26), whose study duration was 6 months. They stated that the finding of decreased adiposity in the male participants in the intervention group was most likely a result of better adherence to the physical activity portion of the intervention and greater intensity of activity, but this observation needs to be evaluated over a longer duration to obtain a more definitive answer.

Most of the reviewed studies included multiple areas of intervention, this integrative review of available current literature points to no specific intervention or combination of interventions as the most beneficial. This observation is supported by Stevens (47) which arise the question is the failure or modest success of the intervention was because of a problem in the content of the intervention approach or with its implementation?

There is strong evidence linking physical inactivity (sedentary behavior) with obesity in children and adolescents (48). In the present review, reduction of sedentary activities was included as a main element in obesity prevention program in three of the reviewed studies $(15,16,37)$. These studies measured the outcomes in term of increasing the reduction of sedentary activities, which adds to the growing evidence that sedentary lifestyles are a concern for all children. 
Table 3: Interventions utilized in childhood obesity prevention

\begin{tabular}{|c|c|c|c|c|}
\hline Subjects & $\begin{array}{c}\text { Intervention: } \\
\text { dietary }\end{array}$ & $\begin{array}{l}\text { Intervention: } \\
\text { physical activity }\end{array}$ & $\begin{array}{c}\text { Intervention: } \\
\text { parental } \\
\text { involvement }\end{array}$ & $\begin{array}{c}\text { Intervention: } \\
\text { healthy } \\
\text { lifestyle education }\end{array}$ \\
\hline Black et al. (2010) & Yes & Yes & Yes & Yes \\
\hline Bruss et al. (2009) & No & Yes & Yes & Yes \\
\hline Caballero et al. (2003) & No & Yes & Yes & No \\
\hline Contento et al. (2007) & Yes & Yes & No & No \\
\hline Donnelly et al. (2009) & No & Yes & No & No \\
\hline Foster et al. (2008) & Yes & No & No & Yes \\
\hline Gentile et al. (2009) & Yes & Yes & No & Yes \\
\hline Gutinet al. (2008) & Yes & Yes & Yes & Yes \\
\hline Haerens et al. (2006) & Yes & Yes & Yes & No \\
\hline James et al. (2004) & Yes & No & No & Yes \\
\hline Jiang et al.(2007) & Yes & Yes & Yes & No \\
\hline Kain et al. (2004) & Yes & Yes & Yes & No \\
\hline Kipping et al. (2008) & Yes & Yes & No & No \\
\hline Marcus et al. (2009) & Yes & Yes & No & No \\
\hline Mauriello et al. (2010) & Yes & Yes & No & No \\
\hline McMurray et al. (2002) & No & Yes & No & Yes \\
\hline Neumark-Sztainer et al. (2003) & Yes & Yes & No & No \\
\hline Paineau et al. (2008) & Yes & No & Yes & No \\
\hline Rodearmel et al. (2006) & Yes & Yes & Yes & Yes \\
\hline Sahota et al. (2001) & Yes & Yes & Yes & No \\
\hline Sallis et al. (2003) & Yes & Yes & No & No \\
\hline Salmon et al. (2008) & No & Yes & No & Yes \\
\hline Sichieri et al. (2009) & Yes & Yes & No & No \\
\hline Simon et al. (2008) & No & Yes & No & Yes \\
\hline Singh et al. (2007) & Yes & Yes & No & No \\
\hline Spiegel \& Foulk (2006) & No & Yes & No & No \\
\hline
\end{tabular}

\section{Limitations}

Despite comprehensive efforts to identify all relevant studies, it is possible that some may have been missed. Because studies considered for inclusion were in English language only, further data might have been revealed from including non-English language studies. In addition, the studies included in this review were conducted in a variety of countries with a diversity of cultures and the degree of their generalizability is uncertain.

\section{Conclusion}

The purpose of this systematic review was to identify the current state of the evidence related to the prevention of overweight and obesity in children and adolescents. The results indicate three areas of emphasis in the literature: community settings as the future focus for addressing the childhood obesity epidemic is strong, prevention as the best option; crucial parental involvement; and no specific intervention or combination of interventions as the most beneficial approach. Health care practitioners must be involved in developing and implementing well-constructed implementation and evaluation studies that build on the limited base of current evidence.

\section{Conflict of Interest Statement}

The authors declare that there are no conflicts of interest. 


\section{Acknowledgement}

No grants were obtained in relation to this paper.

\section{References}

[1] American Academy of Pediatrics: Policy statement: Prevention of Pediatric Overweight and Obesity. Pediatrics 2003, 112: 424-430.

[2] Centers for Disease Control and Prevention (CDC): National Health and Nutrition Examination Survey (NHANES). Overweight among U.S. children and adolescents 2003, Retrieved May 24, 2011, available at: http://www.cdc.gov/nchs/data/nhanes/ databriefs/overwght.pdf.

[3] Lobstein T, Bauer L, \& Uauy R: Obesity in children and young people: A crisis in public health. Obesity Reviews 2004, 5 (1), 4-85.

[4] World Health Organization: Global health risks: Mortality and burden of disease attributable to selected major risks. Department of Health Statistics and Informatics. Geneva, Switzerland, 2009.

[5] Shaya FT, Flores D, Wang J: School-Based obesity interventions: A literature review. Journal of school Health. 2008, 78: 189-196.

[6] Melnyk BM, Small L, Morrison-Beedy D, Strasser, A, Spath, L, Kreipe, R, et al. Mental health correlates of healthy lifestyle attitudes, beliefs, choices, and behaviors in overweight adolescents. Journal of Pediatric Health Care 2006, 20: 401-406.

[7] Puhl RM, \& Latner JD: Stigma, obesity, and the health of the nation's children. Psychological Bulletin 2007, 133: 557-580.

[8] Becky JC: Targeting the Obesity Epidemic in Children and Adolescents: Research Evidence for Practice. Journal of Pediatric Nursing 2011, 26: 503-506.

[9] Trogdon JG, Finkelstein EA, Feagan CW, \& Cohen JW: State and payer-specific estimates of annual medical expenditures attributable to obesity. Obesity (Silver Spring) 2012, 20 (1):214-20.

[10] Lobstein T, \& Millstone E: Context for the PorGrow study: Europe's obesity crisis. Obesity Review 2007,8 (2): 7-16.

[11] Ells LJ, Campbell K, Lidstone J, Kelly S, Lang R, Summerbell C: Prevention of childhood obesity. Baillieres best Practice Res Clin Endocrinol Metab 2005, 19(3):441-454.

[12] Wojcicki J, \& Heyman M: Let's move-Childhood obesity prevention from pregnancy and infancy onward. 2010, Available at: http://www.nejm.org/doi/full/10.1056/NEJMp1001857 on 10th April 2011.

[13] Neumark-Sztainer D., Story M. Peter J. H, Rex J. New Moves: a school-based obesity prevention program for adolescent girls. Preventive Medicine 2003, 37: 41-51.

[14] Marcus C, Nyberg G, Nordenfelt A, Karpmyr M, Kowalski J, Ekelund UA: 4-year, cluster-randomized controlled childhood obesity prevention study: STOPP. International Journal of Obesity 2009, 33(4):408-17.

[15] Contento R, Koch P, Lee H, Sauberli W, Calabrese-Barton A: Enhancing personal agency and competence in eating and moving: Formative evaluation of a middle school curriculum-choice, control and change. Journal of Nutrition Education and Behavior 2007, 39(5): S179-S186.

[16] Bruss MB, Michael TJ, Morris JR, Applegate B, Dannison L, Quitugua JA, Palacios RT, Klein DJ: Childhood Obesity Prevention: An Intervention Targeting Primary Caregivers of School Children. Obesity 2010, 18: 99-107.

[17] Black MM, Hager ER, Le K, Treuth M, DiClemente Y, Gittelsohn J, Magder L, Papas M, Snitker S: Challenge! Health promotion/obesity prevention mentorship model among urban, black adolescents. Pediatrics 2010, 126: 280-288.

[18] Caballero B, Clay T, Davis SM, Ethelbah B, Holy Rock B, Lohman T, Norman J, Story M, Stone EJ, Stephenson L, Stevens J: Pathways: A school-based, randomized controlled trial for the prevention of obesity in American Indian school children. The American Journal of Clinical Nutrition 2003, 78: 1030-1038.

[19] Donnelly JE, Greene JL, Gibson CA, Smith BK, Washburn RA, Sullivan DK, DuBose K, Mayo MS, Schmelzle K, Ryan J, Jacobsen D, Williams S: Physical Activity Across the Curriculum (PAAC): a randomized controlled trial to promote physical activity and diminish overweight and obesity in elementary school children. Preventive Medicine 2009, 49(4):336-341.

[20] Foster GD, Sherman S, Borradaile KE, Grundy KM, Vander Veur SS, Nachmani J, Karpyn A, Kumanyika S, Shults J: A policy-based school intervention to prevent overweight and obesity. Pediatrics 2008, 121(4): e794-802.

[21] Gentile DA, Welk G, Eisenmann JC, Reimer RA, Walsh DA, Russell DW, Callahan R, Walsh M, Strickl S, Fritz K: Evaluation of a multiple ecological level child obesity prevention program: Switch what you Do, View and Chew. BMC Med 2009, 7:49.

[22] Gutin B, Yin Z, Johnson M, Barbeau P: Preliminary findings of the effect of a 3-year after-school physical activity intervention on fitness and body fat: the Medical College of Georgia Fitkid Project. International Journal of Pediatric Obesity 2008, 3(1): 3-9.

[23] Haerens L, Deforche B, Maes L, Cardon G, Stevens V, \& De Bourdeaudhuij I: Evaluation of a 2-year physical activity and healthy eating intervention in middle school children. Health. Education Research 2006, 21(6), 911-921.

[24] James, J., Thomas, P., Cavan, D., \& Kerr, D. (2004). Preventing childhood obesity by reducing consumption of carbonated drinks: Cluster randomized control trial. British Medical Journal, 328, 1237- 1239.

[25] Jiang, J., Xia, X., Greiner, T., Wu, G., Lian, G., Rosenqvist, U., 2007. The effects of a 3-year obesity intervention in schoolchildren in Beijing. Child: Care, Health and Development. 33, 641-646. Available at: http://dx.doi.org/10.1111/j.1365-2214.2007.00738.x

[26] Kain J, Uauy R, Albala Vio F, Cerda R, Leyton B: School based obesity prevention in Chilean primary school children: methodology and evaluation of a controlled study. Int J Obes Relat Metab Disord 2004, 28: 483-493.

[27] Kipping RR, Payne C, Lawlor DA. Randomized controlled trial adapting US school obesity prevention to England. Archives of Disease in Childhood 2008, 93(6):469-73.

[28] Mauriello LM, Ciavatta MH, Paiva AL, Sherman KJ, Castle PH, Johnson JL, Prochaska JM: Results of amulti-mediamultilple behavior obesity prevention program for adolescents. Preventative Medicine 2010, 51: 451-456.

[29] McMurray RG, Harrell JS, Bangdiwala SI, Bradley CB, Deng S, Levine A: School-based intervention can reduce body fat and blood pressure in young adolescents. J Adolesc Health 2002, 31:125-132.

[30] Paineau DL, Beaufils F., Boulier A., Cassuto DA, Chwalow J, Combris P, Couet C, Jouret B, Lafay L, Laville M, Mahe S, Ricour C, Romon M, Simon C, Tauber M, Valensi P, Chapalain V, Zourabichvili O, Bornet F. Family dietary coaching to improve nutritional intakes and body weight control: a randomized controlled trial. Arch. Pediatr. Adolesc. Med. 2008, 162: 34-43.

[31] Rodearmel SJ, Wyatt HR, Barry MJ, Dong F, Pan D, Israel RG, Cho SS, McBurney MI, \& Hill JO: A family-based approach to preventing excessive weight gain. Obesity 2006, 14(8):1393-401.

[32] Sahota P, Rudolf M, Dixey R, Hill A, Barth J, Cade J. Evaluation of implementation and effect of primary school based intervention to reduce risk factors for obesity. BMJ 2001, 323: 1027-1029. 
[33] Sallis JF, McKenzie TL, Conway TL, Elder JP, Prochaska JJ, Brown M et al. Environmental interventions for eating and physical activity: a randomized controlled trial in middle schools. Am J Prev Med 2003, 24: 209-217.

[34] Salmon J, Ball K, Hume C, Booth M, Crawford D: Outcomes of a group-randomized trial to prevent excess weight gain, reduce screen behaviours and promote physical activity in 10-year-old children: switch-play. International Journal of Obesity 2008, 32(4): 601-12.

[35] Sichieri R, Paula Trotte A, de Souza RA, Veiga GV: School randomised trial on prevention of excessive weight gain by discouraging students from drinking sodas. Public Health Nutrition 2009, 12 (2):197-202.

[36] Simon C, Schweitzer B, Oujaa M, Wagner A, Arveiler D, Triby E, et al. Successful overweight prevention in adolescents by increasing physical activity: a 4-year randomized controlled intervention. International Journal of Obesity 2008, 32(10): 1489-98.

[37] Singh AS, Chin A Paw MJ, Brug J, Van Mechelen W. Short term effects of school-based weight gain prevention among adolescents. Archives of Pediatrics \& Adolescent Medicine 2007, 161: 565-71.

[38] Spiegel SA, \& Foulk D: Reducing overweight through a multidisciplinary school-based intervention. Obesity 2006, 14(1):88-96

[39] Waters E, de Silva-Sanigorski A, Hall BJ, Brown T, Campbell KJ, Gao Y, Armstrong R, Prosser L, Summerbell CD: Interventions for preventing obesity in children (review). Cochrane Library 2011, 12:1-212.

[40] Lindsay AC, Sussner KM, Kim J, \& Gortmaker, S: The role of parents in preventing childhood obesity. The Future of Children 2006, 16: 169-186.

[41] Pyle SA, Sharkey J, Yetter G, Felix E, Furlong MJ, \& Carlos Poston WS: Fighting an epidemic: The role of schools in reducing childhood obesity. Psychology in Schools 2006, 43:361-376.

[42] Lown DA, \& Braunschweig CL: Determinants of physical activity in low-income, overweight African American girls. American Journal of Health Behavior 2008, 32(3): 253-259.

[43] Wofford LG: Systematic review of childhood obesity prevention. Journal of Pediatric Nursing 2008, 23(1), 5-19.

[44] Nielsen SJ, Siega-Riz AM, Popkin BM. Trends in food locations and sources among adolescents and young adults. Prev Med 2002: 35: 107113.

[45] Gittelsohn J \& Kumar MB: Preventing childhood obesity and diabetes: is it time to move out of the school? Pediatric Diabetes 2007, 8 (Suppl. 9): 55-69.

[46] Lytle la, Kubik MY, Perry C, Story M, Birnbaum AS, Murray DM. Influencing healthful food choices in school and home environments: results from the TEENS study. Prev Med 2006: 43: 8-13.

[47] Stevens CJ: Obesity Prevention Interventions for Middle School-Age Children of Ethnic Minority: A Review of the Literature. Journal for Specialists in Pediatric Nursing 2010, 15 (3): 233-43.

[48] Joy EA: Practical approaches to office-based physical activity promotion for children and adolescents. Current Sports Medicine Reports 2008, 7(6): 367-372. 